Vol. 3, No. 2 | July - December 2020

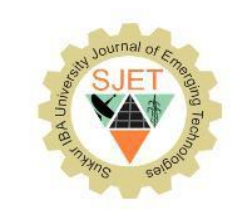

\title{
Nature Inspired Optimization Techniques: A review for FANETs
}

\author{
Shahzad Hameed ${ }^{1}$, Qurratul-Ain Minhas ${ }^{1}$, Sheeraz Ahmed ${ }^{2}$, Asif Nawaz ${ }^{3}$, \\ Junaid Iqbal $^{4}$, Muhammad Shahzad ${ }^{5}$, Naveed Jan ${ }^{6}$
}

\begin{abstract}
:
Flying Ad Hoc Network (FANET) is a revolutionary achievement in the class of ad hoc networks to access areas near calamities. The concept of pilot free system takes place in technological advancement and computations. Networking advancements introduces Unmanned Aerial System (UAS) while the device that makes this system applicable is known as flying drones or Unmanned Aerial Vehicle (UAV). Single UAV may be employed as individual system, but it is more useful when UAVs in a network cooperate with each other. UAV deployments are rapid and pliant that reduces the tradition ad hoc network limitations. UAV have small operating structure and efficient energy life. Advance flight control mechanism makes FANETs differ from traditional ad hoc networks. FANETs can visualized to play key role where humans are in danger or rescue tasks are too dull or dirty. Improving the performance of flying drones leads to ease-of-use for formation of network, high stability and fast deployment. Routing and cooperative task allocation of nodes is complex in ad hoc networks. In contrast of the study of traditional schemes, our paper is based on the nature inspired schemes. This review study in on routing protocols of BeeAdHoc, AntHocNet and Moth-Ant inspired routing. In addition, the overview leads to wild life behavior of Hill Myna and Desert Sparrow Optimization comparative to other biological algorithms is presented in terms of task allocation and rendezvous cooperatively. Biological inspired behaviors trend to know the efficient problem solutions for FANETs.
\end{abstract}

Keywords: FANET; BeeAdHoc; AntHocNet; Moth-Ant inspired routing; HMADSO

\section{Introduction}

FANET is a subclass of VANET that governs the autonomous movement of UAV. The application scenarios in FANETs are forest fire detection, rescue and search operation, patrolling, traffic and urban monitoring, reconnaissance, agricultural management, environmental sensing and relaying network [1]. The multi UAV plays an important role in choosing a networking architecture for best performance. There exist

${ }^{1}$ Department of Electronics, Quaid-i-Azam University, Islamabad, Pakistan

${ }^{2}$ Department of Computer Science, Iqra National University, Peshawar, Pakistan

${ }^{3}$ Faculty of Electronics, Higher Colleges of Technology, Dubai, U.A.E

${ }^{4}$ Department of Mechanical Engineering Quaid-e-Awam University, Larkana-Campus

${ }^{5}$ Department of Electrical Engineering and Technology, MNS UET, Multan, Pakistan

${ }^{6}$ Department of information engineering technology University of Technology Nowshera Corresponding Author: shahzadhameed01@yahoo.com 
different networking architectures proposed and emerged. The basic one is the centralized link communication between UAVs and single ground station in a fixed topology in which a ground station is responsible for creating the communication between UAVs as well as their coordination movements. The usage of a Ground Control Station (GCS) might results in traffic congestion that accordingly influences the system functionality [2]. QoS in the FANET is very important in case of emergency services and data packet delivery ratio and delay are also important factors in FANETs domain.

Flying Drones have optimal capacities for covert operations, intelligence, surveillance, target acquisition, preventive noise pollution and reconnaissance. UAV can perform long and high precision operations for civilian, commercial and military applications. UAVs are designed for long endurance despite harsh weather conditions. Due to its high Detection, Recognition and Identification (DRI) capabilities and resistance to jamming and interference, it is ideally suited for site protection and civil security. FANETs involve fast changing topology, frequent network partitioning (nodes leave or join) and three-dimensional movements. They have self-organizing, selfhealing abilities which leads the life of wireless network. Protocols on the other hand can run in different layers to handle dynamic entering and leaving of nodes. Each flying UAV in FANETs considers as a router to achieve real-time effective communication with advance features of perfect actuators, latest software and best loop principles. With temporary wireless communication setup, only FANETs provides quick and real time rescuing service and also provide on demand public safety services. Fig.1 shows the design system of FANET that can increase the rescuing chances in natural disaster environments.

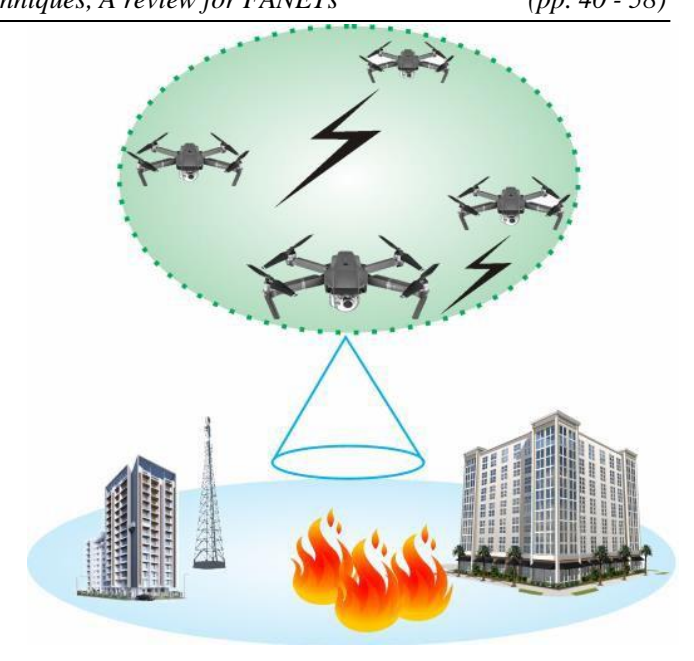

Fig. 1. FANET in disaster environment

Unpredictable event like wildfires occurs in timberland areas (forests and prairies) and could also happen in urban areas (buildings and homes). Three possible network architectures proposed for the multiUAV system are satellite, cellular, ad hoc and solves or alleviates the problems in direct link approach [3]. Networking architecture in FANET established the wireless network among UAVs and each UAV can perform as a relay node, so that works together in a manner that to forward the packets until it reaches to the target. There are two segments involves according to the communication type. The first one is called "ground or satellite segment" where some UAVs directly communicate with base station or via satellite and it is also known as UAV-to-infrastructure communication. "Aero segment" is the second segment of communication in which the UAVs do not have any direct links with the ground station and they are connected with each other. This communication type is known as UAV to UAV communication. It is important to emphasize that changing in the orientation from using one UAV to several small UAVs there is a need to develop new networking technologies among UAVs. FANET is within that context considered as a popular 
technology for a communication networking for multi UAV to extend the operational ambit and ranging, it is also capable of providing quick and reliable response time [4].

The rest of the paper is organized into different sub sections and are describe as follows. Section 2 presents literature. Section 3 explains the categorization of FANET routing protocols. Section 4 presents the biologically inspired algorithms. Section 5 presents the simulation analysis of ACO, BCO, Moth-Ant and HMADSO. Section 6 presents the simulation characteristics of these algorithms and at the end this review paper is concluded in section 7 .

\section{Related Work}

Routing and handling the resources efficiently among the ad hoc nodes and their cooperative behavior are the key tasks in the field of ad hoc networks like FANETs. Routing and task allocation can be achieved by picking examples from natural world. Based on social behaviors of animal, biological world trend to open up the inspiration interest from wild life. The researchers have been modifying the traditional algorithms to enhance the life time of network, stability period for reliable communication and to reduce path loss and end to end delay. The selection of protocol for routing and algorithm for cooperative task allocation are the complex task in FANETs and it depends on the application requirements. All these are depending on total users and their mobility level. The algorithms for routing are used for packet data delivery and are applied to find the best route by mean of secure as well as shortest route for packet sending. Now for optimal routes, metrics are defining the selection criteria which may include information of the cost of communication, power consumption, delay, bandwidth, route length (steps number), reliability etc.

Swarm Intelligence (SI) so called swarm algorithm is used when external management, coordination and control are not available to solve the complex dynamic problems. Bio-inspired protocols AntHocNet, BeeAdHoc and moth-ant inspired routing are efficient in terms of routing as compare to the traditional routing algorithms. Further, the algorithm for distribution of different task among nodes to utilize the available nodes efficiently, HMADSO is discussed. The concept of algorithm based on biologically inspired in nature is used for problem solution of complex optimization in FANET. Nature inspired behavior analyzed by authors [5] and presented the biological concept of honey bee in which bees of the hive categorized into three main groups: i) Employed bees ii) Onlookers iii) Scouts.

Authors in [6] implemented a bee algorithm for Mobile Ad hoc Networks (MANETs) for peer to peer file searching and explore the bee behavior. Scouting is the first stage which can be further divided into two scouting stages the first one is forward scouting and the second stage is backward scouting. The role of the forward scouts is to scrutinize the network for the destination node. Information is carried by forward scout that consist of four categories: Scout ID, Source node ID, Minimal residual energy and Number of hops.

A moth-inspired routing algorithm for mobile networks is presented in [7] to improve the communication performance. This protocol can reduce the packet loss and improve the throughput under certain conditions. Further, it has choice to know the non-optimal routing path and analyze to reduce the delay and network overhead. Some routing protocols of other kinds of ad hoc networks have been modifying for applicability in new ad hoc family (FANETs). These routing protocols are classified into six different categories [8]. FANETs are high speed data transfer, high nodes mobility and hence not easy to find the universal problem solution of routing and task allocation in FANET. Categorizations of routing protocols for FANETs are describe as follow. 


\section{Catogarization of FANETs Rouitng} Protocols

Categorization of routing protocols and its applicability in FANETs are describes as follows 1) Static Routing Protocols 2) Proactive Routing Protocols 3) Reactive Routing Protocols 4) Hybrid Routing Protocols 5) Geographic/ 3-D Routing Protocols 6) Hierarchical Routing Protocols.

\subsection{Static Routing Protocols}

Static type protocols hold the static tables, these tables are computed and laden once at the starting of time operation and cannot be updated during task operations. Static protocols are not scalable and cannot handle the fast-dynamic topology changes in FANET but useful in academia interest.

\subsubsection{Data Centric:}

Data centric routing presented depended on content of data and can be used for one to many communication models. This is best for cluster topologies where broadcasting can be done by Cluster Head $(\mathrm{CH})$ to other nodes in the cluster. $\mathrm{CHs}$ are responsible to extend the reliable communication distance.

\subsubsection{Multi Level Hierarchical Routing (MLHR):}

Based on cluster topology MLHR proposed in [9] and solved the large-scale scalability problems in vehicular networks. In FANET multiple UAVs can be grouped to form clusters in which $\mathrm{CH}$ has the ability to link outside cluster. $\mathrm{CH}$ is responsible to disseminate data to another UAV in cluster. In FANET speedy change of $\mathrm{CH}$ enforce large overhead in network.

\subsubsection{Load Carry and Deliver (LCAD):}

LCAD proposed in [10] in which node at the ground is responsible to pass the required information to UAV which securely carry it to destination. LCAD has higher throughput but data delivery delay is longer due to use of single UAV. Maximum throughput and ensure the security are its objectives.

\subsection{Proactive Routing Protocols}

Proactive Routing Protocols (PRPs) contains the table of route knowing the information of network nodes. These protocols store the route data. In PRPs, nodes maintain its table up to date and contain only latest data of the routes. Data is transmitted by sharing control messages (HELLO, TC) in it and routing procedure is performed by each node without any direction from others. It is important in FANET to maintain the tables up to date at the time of topology changes. On the other hand, overhead and large bandwidths are main limitations in it.

\subsubsection{Optimized Link State Routing (OLSR):}

OLSR is the most adaptive routing algorithm proposed in [11] for ad hoc networks. At startup, routes are set in it for all destinations and then by property of proactive routing, nodes within the network contain the updated data of the routes. These nodes are responsible to share the topology information regularly to its adjacent nodes by broadcasting the link state costs using flooding strategy. Recently some extensions are developed to utilize the features of this algorithm in different application.

\subsubsection{Directional Optimized Link State \\ Routing (D-OLSR):}

D-OLSR is an extension of OLSR and proposed by authors in [12]. It differs from OLSR in selection of multipoint relays. Node in a network measure the destination distance by the help of route table and when found the long distance to the destination it will go on directional antenna thru D-OLSR and when the distance to the destination found smaller than it will go back to OLSR and performs Omi directional antenna. D-OLSR has advantage of less end to end delay than OSLR. 


\subsubsection{Multipath Optimized Link State Routing (MP-OLSR):}

The routing MP-OLSR is basically based on OLSR and is proposed in [13] to improve efficiency of data transmission using multipath.MP-OLSR is an application layer protocol. This protocol obtains extensibility by employing different link metrics and cost function with flexibility. In FANET the applicability of MP-OLSR guarantees the Qos for data transmission and in addition, to improve QoS loop detections and route recovery implemented in MP-OLSR.

\subsubsection{Destination Sequence Distance Vector (DSDV):}

Authors in [14] proposed DSDV that used the sequence numbers to avoid the loops and to trace the best route. Based on Bellman Ford algorithm, DSDV is proposed and uses with minor adjustment of ad hoc networks. Two types of packet updates are available in it. The first is full dump and second is incremental. At the time of topology changes the incremental dump packets are sent only. Although DSDV slightly reduce the network overhead but large overhead is there because of periodic updates and required large bandwidth for update process which is necessary in topology changes.

\subsubsection{Fisheye State Routing (FSR):}

FSR is a class of PRP and authors proposed it in [15]. FSR reduce the overhead by mean of more frequent small table update to the adjacent nodes. The idea for such a case is known as fisheye scope. One of the main disadvantages of this routing is inaccuracy of network where nodes are high mobility and dynamic topology. Similarly based on PRP, Border Gateway Protocol with Mobile Extension (BGP-MX) enables inter domain communication in Air Born networks scenario. It allows high mobility network to stay connected to each other [16]. Based on ADS-B setup for aeronautical ad hoc network is proposed in [17]. It is a PRP and a topology aware protocol, the aim of this protocol is to get rid of the traditional routing beaconing and presented the velocity base next hop selection metric. The improvement of cognitive transfer ratio and reduce the network overhead can be seen effectively.

\subsection{Reactive Routing Protocols}

Reactive Routing Protocols (RRPs) are commonly known as on demand routing protocols and these are designed to reduce the PRPs overhead. In RRP a route is stored in a node when it communicates with each other. Further, nature of on-demand assures no periodic messaging for connectivity. Due to this property RRPs are bandwidth efficient. The process to tracing routes can consume long time and caused high latency. The source and the hop by hop routing are the two cases of RRPs. Source to destination address is contain in the packet and based on this information intermediate nodes can only forward packets in source routing. Secondly, the destination and next hop address contains in second type of routing (hop by hop). In this case intermediate node maintain routing table to only forward data.

\subsubsection{Dynamic Source Routing (DSR):}

Dynamic Source Routing [18] in RRP and usually designed to use in multi hop networks. It permits networks to be self healing, self organizing without requirement of any existing infrastructure. DSR is an ondemand routing and scale automatically. The mechanism of route discovery and route maintenance in DSR achieved efficiently. It has advantage to allow choice from many routes to any destination like load balancing.

\subsubsection{Ad hoc On Demand Distance Vector $(A O D V)$ :}

Authors proposed AODV in [19]. It is a class of RRP using hop by hop phenomena and can easily adjust in high mobility and dynamic change scenario. It has lower overhead and it can specify unicast routes to destination. Next hop information in the network corresponding to each data transmission contain by source and relay nodes. These data packets have only 
destination IP address which overcomes the overhead. To extending standard routing algorithm of Open Shortest Path First which is widely used in wired network the extension MANET Designated Routers (OSPF-MDR) is presented for effectively used in wireless [20]. Dynamic Mobile Ad hoc Networks On demand (DYMO) routing protocol presented in [21] effective route discovery and route maintenance are the goal of this protocol.

\subsection{Hybrid Routing Protocols}

Hybrid Routing Protocols (HRPs) over comes limitations faced in PRPs and RRPs i.e. reduce the overhead caused by control messages and decrease the latency comes from startup route discovery process respectively. These types of routing protocols are suitable for large network. In HRP, network is categorized into two different routing zones i.e. intra zone routing and inters zone routing. Proactive routing can be performed by using intra zone routing while reactive routing approach using inter zone. Hybrid routing set the scheme according to the network properties and effective for MANETs. However, nodes behaviors in FANET make it complicated to maintain the information accordingly. It is hard to implement in dynamic networks.

\subsubsection{Zone Routing Protocol (ZRP):}

Using a concept of zones, authors proposed Zone Routing Protocol (ZRP) [22]. Zones are established by nodes within the entire network. Using approach of proactive, intra zone routing maintain routes while data packets are sent out side by inter zone and using reactive approach to maintain routes. This zone based HRP improve the network efficiency in terms of reactive mechanism and also helpful to improve the quality route discover in terms of proactive maintenance.

\subsubsection{Sharp Hybrid Adaptive Routing Protocol (SHARP):}

SHARP [23] protocol finds equilibrium between reactive and proactive routing and proactively set the degree to broadcast the route information and also set the degree to discover the route reactively. The working of reactive and proactive routing protocols varies with network properties and one protocol outperforms the other in different network scenario. This protocol is efficient to reduce the overhead, bounding loss rate and effectively handle the jitter.

\subsubsection{Temporarily Ordered Routing Algorithm (TORA):}

The algorithm namely TORA is proposed in [24]. For multi-hop networks, this algorithm is a family of HRP and routes specifically maintain data knowing the adjacent nodes. The purpose of this protocol is to handle the broadcasting of control messages in high mobility scenario and reduced the impact of topology changes. The protocol TORA does not follow the shortest route but a longer route is often adapted to reduce the overhead effect.

\subsection{Geographic 2-D and 3-D Protocols}

Geographic schemes consider geographic position of nodes and known the information about nodes. The source in geographic model knows the geographical place of the node and messages are sent to the destination without route discovery. Many routing strategies for 2 dimensional networks have been developed by adapting planar geometry. The main technique use for this model is greedy forwarding. The main purpose is to decrease the number of hops and contain only local information. In this way every node pass the data to the node adjacent to the destination. In FANET application it may be more convenient by using 3 dimensions. High dynamic topology changes and high mobility need the 3-D environments to scale the parameters.

\subsubsection{Greedy Random Greedy (GRG):}

GRG forwarded the message greedily till local minima are encountered. Authors in the paper [25] analyzed simulation results of five recovery algorithm and compared them to find the best one. Based on 
random walk are on surface, on dual and on graph further are namely bounded DSF on spanning tree and bounded flooding. Random walk schemes are best for denser networks while DSF on spanner approach shown effectual performance for sparse networks. GHG is acronym of Greedy Hull Greedy protocol presented in [26] for routing on the hull to escape local minima. The researchers have proposed a protocol namely Partial Unit Delaunay Triangulation (PUDT). The purpose of this protocol is to split up the network into amount of close sub spaces to handle the local recovery procedure.

\subsubsection{Greedy Perimeter Stateless Routing (GPSR):}

Greedy Perimeter Stateless Routing (GPSR) presented by authors in [27]. Its purpose is to use the data packet and position of the routes to enable data packet forwarding decisions. These decisions are depending on only local information of adjacent in the network topology. Whenever greedy forwarding is not possible, this algorithm recovered by routing about the circumference of the area. Using the property containing only local topology state, this protocol scales efficient in per router state than shortest path. In high mobility topology changes, GPSR is capable to use local information for tracing the appropriate routes quickly.

\subsubsection{Location Aided Routing (LAR):}

Authors in [28] presented the concept to utilize location information. Idea based on global location system it presented to improve the working of LAR protocol for ad hoc networks. By obtaining local information, this routing algorithm limits the search for updated route to a minor request zone of a ad hoc network that caused to decrease number of routing messages and reduce the overhead.

\subsubsection{UAV Search Mission Protocol (USMP):}

Protocol for UAVs proposed in [29] for 2-D searching grid. By combination a searching property like search protocol and
USMP, inter UAV link having geographic routing produce to improve the search in efficient way like complete search, UAV distance, and the minimization of UAV direction changes. Further, to improve the search efficiency messages are geographically routed by determining where search position updates its search decisions.

\subsection{Hierarchical Protocols}

Hierarchical protocols are the set of routing solutions. These protocols are proposed for problem solution of scalability and overheads. Hierarchical protocols are one of the possible routing solutions in FANET. Different hierarchy of protocols reduces the network congestion. The hierarchical protocols divided the plan in different levels and routing is performed in these levels. The concept of Cluster develops for different target operation areas. Each cluster in the selected region has a principal cluster called Cluster Head $(\mathrm{CH})$ and each node in the clusters are within the range of $\mathrm{CH}$ for reliable transmission. $\mathrm{CH}$ provides link outside the region with other network for large scale transmission.

\subsubsection{Landmark Ad Hoc Routing (LANMAR):}

Novel based routing namely Landmark Ad hoc Network (LANMAR) presented in [30]. This protocol is achieved by combining the characteristics of landmark and FSR routing for ad hoc networks. A data packet within the entire network initially moves in Landmark behavior for long distance in terms of division of groups of nodes. In meanwhile when it got near to destination, eventually shifted to Fisheye for appropriate route provider. Experimental analysis of LANMAR results scalable and effectual for large routing ad hoc scenario where group mobility exists. Distance Routing Effect ALGORITHM for Mobility (DREAM) proposed in [31]. The proposed protocol mainly develops from two novel observations of distance and position information. Routing table updated with position information of a 
distance function by breakup nodes without disturbing the routing accuracy.

Table I summarizes the classification of protocols and its applicability for FANETs as discussed above.

Table I. Classification of Protocols

\begin{tabular}{|c|c|}
\hline $\begin{array}{c}\text { Classification of } \\
\text { protocols }\end{array}$ & Protocols type \\
\hline Static & $\begin{array}{c}\text { Data-Centric [4] } \\
\text { MLHR [9] } \\
\text { LCAD [10] }\end{array}$ \\
\hline Proactive & $\begin{array}{c}\text { OLSR [11] D- } \\
\text { OLSR [12] MP- } \\
\text { OLSR [13] } \\
\text { DSDV [14] } \\
\text { FSR [15] } \\
\text { BGP-MX [16] } \\
\text { ADS-B [17] }\end{array}$ \\
\hline $\begin{array}{l}\text { On-Demand/ } \\
\text { Reactive }\end{array}$ & $\begin{array}{c}\text { DSR [18] } \\
\text { AODV [19] } \\
\text { OSPF-MDR [20] } \\
\text { DYMO [21] }\end{array}$ \\
\hline Hybrid & $\begin{array}{c}\text { ZRP [22] } \\
\text { SHARP [23] } \\
\text { TORA [24] } \\
\end{array}$ \\
\hline $\begin{array}{c}\text { Geographic/ } \\
\text { 3-D }\end{array}$ & $\begin{array}{c}\text { GRG [25] } \\
\text { GHG [26] } \\
\text { GPSR [27] } \\
\text { LAR [28] } \\
\text { USMP [29] }\end{array}$ \\
\hline Hierarchical & $\begin{array}{c}\text { LANMAR [30] } \\
\text { DREAM [31] }\end{array}$ \\
\hline
\end{tabular}

\section{Biological Inspired Algorithms}

It is complex to declare someone universal problem solution of the routing and task allocation in FANET. In [32] researchers used the differential geometry to design the guidance system with a capability of targets tracking and rendezvous with UAVs. The concepts of velocity factors and targets of UAVs is presented by researches with a mathematical solution. In research paper [33] the design for UAVs of a cooperative localization mechanism is presented by using the non-parameterized belief algorithm. The authors consider dynamic variables for task allocation in terms of position of UAVs in a predefine ambit. Authors in [34] proposed a framework using UAVs for cooperative searching. Reliability and movement planning are used as determination parameters for cooperative task allocations while governing task performed by the framework with motion planning and their control. Unlike traditional networks the study of swarm organizing can be develop from nature. The nature based biological inspired algorithms changed the model of networking in the field of biological behavior for optimal solution.

Structure of topology algorithm on Particle Swarm Optimization (PSO) for FANET proposed in [35]. Construction of topology ensured the operation of end to end communication between the Ground Control Station (GCS) and UAV. Every UAV perform the assign job through optimizing the position of relay UAVs. Proposed algorithm is compared with random structure topology algorithm and as a result, instead of choosing an optimization theoretic approach, PSO algorithm got preferred to structure a FANET topology.

\subsection{Bee Colony Optimization Algorithm}

The best adaptive algorithm is a Bee colony algorithm when deal with topology changes. Bee optimization algorithm differs from some conventional routing algorithms used for routing in telecommunication networks. Alike to other swarms' algorithm, algorithm of bee started to collect the data and transmitted to other nodes. Fig. 2 shows the bee colony behavior diagram. By nature, the bee hive operational principles are pre-define for allocation of responsibilities among the bees. In figure searching begins by employed bee for a nectar source while in the hive, the other bees (onlookers) are remain and data received only from the employed bees by attracting special "waggle dance". This waggle dance devolves on the sun location. The motion of the bee strongly indicated where precisely food is located. Finally scout bee randomly search for new nectar sources. 


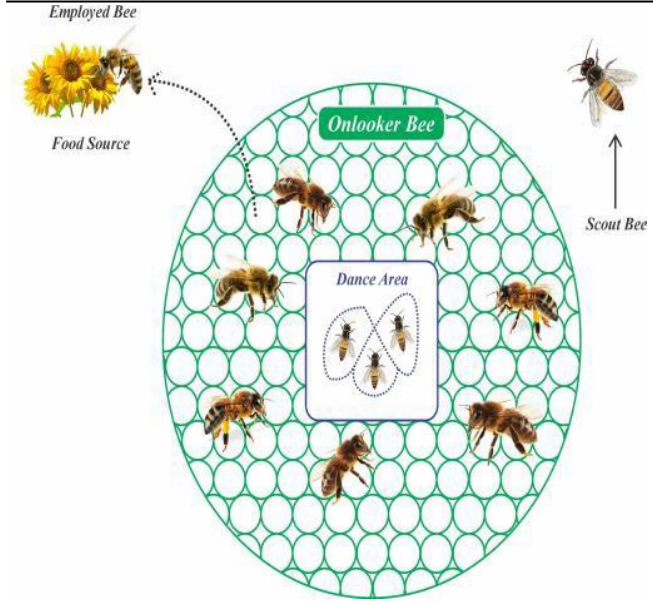

Fig. 2. Bee colony behavior in wild life

In [36] checking the scout characteristics of every visited node the conclusion of route efficiency can be draw by the algorithm. The probabilistic calculation is maintained by probability distribution table. This is complex procedures which require several verification steps. Such things happen like, if more than one forward scout received by a node. It confirmed it first that either the scout has arrived before or not with help of its ID, next important unit is forager-bees come in action after culmination of scout process and route conclusion. The foragers transfer the information like a real bee carries the food. The number of foragers played an important role for the route calculation using dance formula phenomena and then phase of resource foraging started. The procedure of data transmittal is quite complex whenever information is transmitted from one node to the other node and hence vary the amount of forager-bees subject to network condition.

\subsection{Ant Colony Optimization Algorithm}

Comparative to the traditional protocols for wireless ad hoc network, ACO is differs due to its biological environments. Fig. 3 shows the behavior of ACO algorithm with equal task distribution. The process of food search by ant in wild life can be compared with on demand or reactive component on event calling in communication session. While by definition, proactive component operates periodically to maintain and bring the latest data on the existing routes and to improve connection during communication period. The main difference between these two components is reactive component comes into action only when the existing known routes comes faulty. So when deal with ant algorithm in nature it is just betterment of the route between hill ant and food.

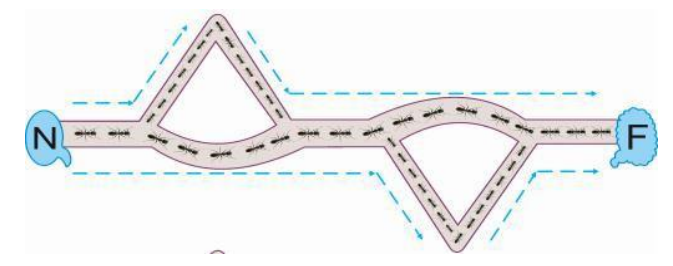

Fig. 3. Initial equal task distribution

In ACO algorithm formation of possible channels between source and destination using ant behavior or control data packets in communication that gather the routing information using iterative sampling. Fig 4 shows the behavior of ant for searching the food in different possible ways in the field. Fig 5 shows the optimized route between nest and food which is the shortest one. The technique of creating protecting routes during link break the procedure of route repair is used in FANET for efficiency and reliability of data. All the decision comes from source node while intermediate nodes in ACO algorithm made no decisions in routing. Intermediate nodes play an important role and store the pheromone tables. In [37] the analysis did perform to know the working and draw nodes capable for decisions making. The foragers did unify and built a single swarm then it come back to the source after completion of appraise. Same ID of the route for forager can help to performed this. Determinations of number of routes at the time of come back and ineffective are eliminated from route tales. 


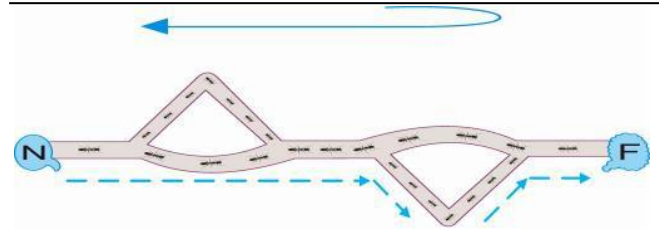

Fig. 4. Following the field searching food

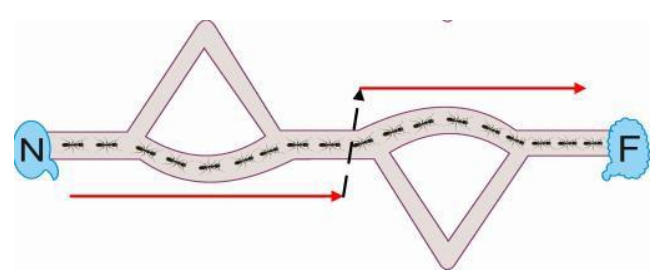

Fig. 5. Optimized route between nest and food

\subsection{Moth and Ant Algorithm}

Peculiar trajectory of the male for light source is due to searching and then it observed the direction of light origin with its natural capability of piercing thermal sensing to locate a direction. The natural behavior of the moth-ant shows in Fig. 6. Initially it follows a straight line by using dive speed to reach the target quickly. In second step when it senses that the target is near, it slowdowns the speed and cautiously fly in the direction of target and follow the zigzag curve. Finally, the moth realized that the source of light is closer than by nature it adopted the circular fly to lock the source. Now this model deal in low level routing of the network based on the trail of ant pheromone for maintenance and used to follow the trajectory. Hence this scenario minimizes the delay. Similarly, ants use striking chemic to put down the trajectory to leave the path to their partner where they can promptly find the source of food such a feature refer to the time decay property.

Three levels of networks for routing protocols are high level network, low level network and cross level presented in [38] and exploited the directional antennas for multi beam of hierarchical network. Considering long space links and high rate of communication a fence routing protocol exploit the effectual benefits in high level of multi beam antennas network with higher throughput and lower delay in competitive to traditional protocols. Moth-inspired routing is capable for low level network with high density and short distance. Low rate link overcome the singular mobility of sink in terms of less packet loss and higher throughput than traditional mobile routing protocols. Moth and ant behavior have high throughput and low latency. In level routing, this protocol ensures the approaching chance to the commander node situated in high level network using cross level technique. The concept of cross level is in order to deliver packet from low level network to the node in high level network.

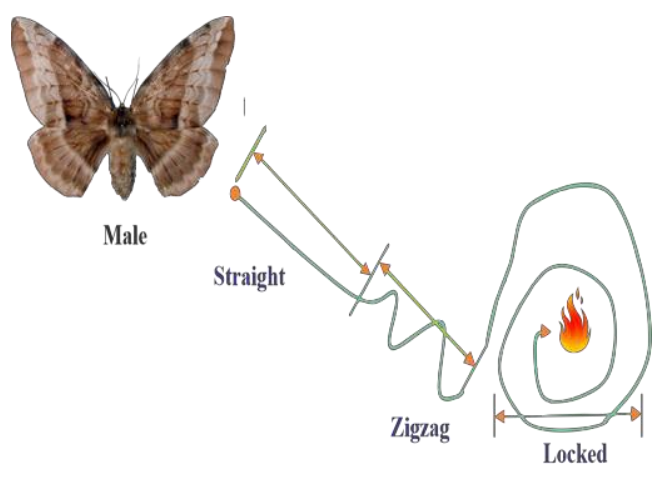

Fig. 6. The behavior diagram of male moth

\subsection{Hill Myna and Desert Sparrow Optimization (HMADSO)}

A new optimization algorithm presented in [39] based on the natural feature for HMADSO. Hill Myna optimization provided effectual route rendezvous. Optimization based on desert sparrow optimization provided task allocation cooperatively. Localization based on desert sparrow can obtained from cooperative rendezvous. Rendezvous permitted suitable solution for the cooperative network formation with high mobility nodes. Task distribution cooperatively permitted non-redundant cooperative task treatment among nodes. Both these optimization algorithms are 
complementing to each other and hence, decrease the spatial complexities and time related to cooperative problems in FANET.

\subsubsection{Hill Myna optimization for cooperative rendezvous:}

The Hill Myna in bio-inspired is similar to UAV in FANETs. Hill Mynas are species and have characteristic of common idiom within the range of $15 \mathrm{~km}$. The idiom range of the Mynas in nature is similar to the radio range of the nodes in FANET. The route discovery between UAV is based on the characteristic of the common idioms. Based on its ability for vocal identification it allowed the identification of all the similar species in the entire ambit. By natural ability, these birds do not replicate the vocal qualities which become appropriate and non-equivocal representation. This brings added to free from danger characteristic of hill Myna optimization.

Mynas are sound, clever and precise learning characteristics that added into its lineament. This brings to perform a basic organizing for optimized algorithm. Usual dialect feature of this bird helps out the representation of adjacent neighbors in the allocated area for each Myna. Idiom other than this in the swarm or network distinguishes the availability of another cluster. Cluster organizing is carried out upon this vocal representation and idiom strength in a swarm. This precise choice of cluster controller it coordinated the motion and communication guaranteed among the birds.

\subsubsection{Desert Sparrow Optimization for cooperative task allocation:}

As family of bird, Desert Sparrow has special characteristic of defenders, attackers and protectors. In addition, the capability of this bird permitted the concerted nest building, concerted searching, and identification of neighbor. Defenders action are to protect the other birds from a threat while action of counter attackers are performed by attackers and finally stay in the swarm with the younger strength and these are neither attackers nor defenders and are known as protectors. Biologically inspired coordination of these birds in various tasks can perform cooperatively. Overlapping and ambiguity is reduced by precisely assigning the task distribution and cooperative task allocation among them. During cooperative task operation, each bird knows the position and present level thru which the process is performed by it. Hence, during cooperation the feature of making localization is important. Desert sparrows are intent at resource distribution rather than utilizations. Desert Sparrow has a predefined sight range that assists them in identification of attackers. Further, protection against opponent creatures, they make them enable to make decisions regarding defense. Applying the abilities of desert sparrows, algorithm of cooperative task allocation has been designed. This is actually the integration with hill Myna optimization. Global problem in the field of FANETs are task allocation and rendezvous are solved by this algorithm effectively. Dependency of route tracing of each desert sparrow and their control motion optimized in this algorithm. Based on S-DAC principle this optimization algorithm by mean of Survey, Decision, Awareness, and Cooperate. Usually this algorithm is a novel concept of understanding between nodes. Following necessary steps are required for task operation. At first it started with an initial survey which enables them for courtly decision further, awareness of these decisions to the others are made to secure the swarm and at last cooperation is made for all components to build mutual control. Table II summarizes the algorithms as discusses above and their features and limitations in FANETs in comparison of traditional schemes. 
Table II: Features and limitations of BeeAdHoc, AntHocNet, Moth-ant inspired and HMADSO

\begin{tabular}{|c|c|c|}
\hline Name & Features & Limitations \\
\hline $\begin{array}{l}\text { Bee Colony } \\
\text { Algorithm [36] }\end{array}$ & $\begin{array}{l}\text { - Better throughput than DSR and DSDV. } \\
\text { - May be used in packet routing in FANET. } \\
\text { - BeeAdHoc overhead increase in proportion } \\
\text { to the nodes amount. } \\
\text { - Most adaptive algorithm in rapid topology } \\
\text { change FANETs. } \\
\text { - Quickly adjust to the changes with the rate } \\
\text { increase and show the best throughput } \\
\text { values. } \\
\text { - Nodes increases cause the increase in the } \\
\text { throughput. } \\
\text { - Alike DSDV and DSR this algorithm is good } \\
\text { as routing. }\end{array}$ & $\begin{array}{l}\text { Longer delay due to search of } \\
\text { new destination. } \\
\text { - Only slightly effect on } \\
\text { throughput. } \\
\text { - Somehow inferior to AODV } \\
\text { and DSR. } \\
\text { Not good for task optimization } \\
\text { in FANETs. }\end{array}$ \\
\hline $\begin{array}{l}\text { BeeAdHocAnt } \\
\text { HocNet } \\
\text { protocols [37] }\end{array}$ & $\begin{array}{l}\text { - AntHocNet protocol performs efficient in } \\
\text { FANETs. } \\
\text { - This protocol has fine scalability. } \\
\text { - Throughput increases as number of nodes } \\
\text { increases } \\
\text { - More effective in contrast to other } \\
\text { biological algorithms. }\end{array}$ & $\begin{array}{l}\text { - Not optimal in terms of } \\
\text { throughput. } \\
\text { - Only used for data delivery } \\
\text { routing. } \\
\text { Not easy to declare universal } \\
\text { routing problem solution. } \\
\text { Routing overhead increases as } \\
\text { node quantity increases. } \\
\text { Lower throughput of DSDV } \\
\text { when the nodes amount } \\
\text { increases. }\end{array}$ \\
\hline $\begin{array}{l}\text { Moth-ant } \\
\text { routing } \\
\text { protocol }[38]\end{array}$ & $\begin{array}{l}\text { Significant throughput as compares to } \\
\text { traditional protocols. } \\
\text { - Smaller updating period brings a higher } \\
\text { throughput. } \\
\text { - Validate the cross-level routing scheme. } \\
\text { - It has better scalability. } \\
\text { - Moths inspired at low speed achieve good } \\
\text { performance. } \\
\text { - Sink speed of moth increase as a result in } \\
\text { increase in throughput in low level routing }\end{array}$ & $\begin{array}{l}\text { - Not good for resource } \\
\text { distribution. } \\
\text { Not optimal throughput } \\
\text { comparative to AODV and } \\
\text { DSR. } \\
\text { - High packet loss when deal } \\
\text { with cross level routing. } \\
\text { Task optimization problem } \\
\text { remain for FANET. }\end{array}$ \\
\hline HMADSO [39] & $\begin{array}{l}\text { HMADSO has best performance associated } \\
\text { with UAVs. } \\
\text { - Good for cooperative task allocation in } \\
\text { FANET. } \\
\text { - It can obtain cooperative task allocation and } \\
\text { cooperative rendezvous. } \\
\text { - Time and intricate of cooperative problems } \\
\text { in FANETs are reduced. } \\
\text { - In terms of task handling it is better than } \\
\text { ACO and BCO. } \\
\text { - It deals better when nodes are increased in } \\
\text { the network. } \\
\text { - Achieving better cognitive ratio. }\end{array}$ & $\begin{array}{l}\text { - It has high network latency. } \\
\text { Cannot declare universal } \\
\text { problem solution of task } \\
\text { allocation. }\end{array}$ \\
\hline
\end{tabular}




\section{Simulation Analysis of Bio Inspired Schemes with Traditional Schemes}

\subsection{BeeAdHoc, AntHocNet:}

In order to measure the performance of routing protocols, necessary metrics are throughput, end-to-end delay routing overhead. Routing is a complex problem in FANETs, biological approaches have been describing the routing problem solution for FANETs. Packet data routing delivery in FANETs may be used biological inspired algorithms ACO and BCO. These are only for routing purpose but not suitable and efficient for task optimization. Increase in the number of nodes within the targeted area may increases the throughput of the protocols. $\mathrm{BCO}$ is the most adaptive algorithm in topology change situation especially its rapid adjustment with the change in the rate increase and show the best throughput values. BCO has longer delay because of searching the new routes to the destination. AntHocNet performed efficiently in FANETs environment. This protocol has fine scalability and same as BCO the throughput of this protocols increase as number of nodes increases. When the node rate slightly changes in rate, the heavy load due to routing overhead with increase in node mobility remains the throughput level at same position. ACO and BCO methods appear more effectual in comparative to other biologically inspired methods. BCO and ACO are used for data delivery and routing but not good for task optimization problems. Comparison characteristic of BeeAdHoc, AntHocNet with traditional protocols (AODV, DSDV, DSR) shows in table III.

Table III. Comparison characteristic of BeeAdHoc, AntHocNet with traditional protocols.

\begin{tabular}{|c|c|c|c|}
\hline Protocols & End-to-End delay & Throughput & Overhead \\
\hline BeeAdHoc & Inferior to AODV and DSR & High & Low \\
\hline AntHocNet & Less than AODV & High & High \\
\hline AODV & High & High & High \\
\hline DSDV & Low & Low & High \\
\hline DSR & Low & High & High \\
\hline
\end{tabular}

Routing overhead increases for all the protocols only when number of nodes in the network increases. DSDV and DSR are under small load when nodes mobility has increased with respect to routing overhead. Longer delay in BeeAdHoc relative to the other protocols due to unnecessary finding of new routes to the destination. An advantage over the other protocols by the throughput became it most popular. Better throughput achieved from BeeAdHoc than DSDV and DSR, but has longer delay.

\subsection{Moth-Ant Inspired Routing}

Moths inspired at a low speed can achieve better performance due to construction of non-optimal path comparative to traditional mobile routing. Moth routing has significant throughput as compare to traditional protocols. Sink speed of moth 
increases as a result in increase in throughput with cross level routing. Moth-ant scheme in low level routing. Ant- inspired validate the compares with traditional schemes is shows in cross-level routing scheme. It has also better table IV. scalability but has high packet loss when deal

Table IV. Comparison characteristic of Moth and Ant with traditional schemes.

\begin{tabular}{|l|c|c|c|c|}
\hline Protocols & Latency & Throughput & Delay & Packet loss \\
\hline $\begin{array}{l}\text { High level network (Fence } \\
\text { routing protocol) }\end{array}$ & Low & High & Low & Low \\
\hline $\begin{array}{l}\text { Low level network (Moth } \\
\text { inspired routing protocol) }\end{array}$ & Low & High & Low & Low \\
\hline $\begin{array}{l}\text { Cross level network (Ant } \\
\text { inspired scheme) }\end{array}$ & Low & High & Low & Low \\
\hline & & & & \\
\hline
\end{tabular}

TABLE. V. Comparison characteristic of HMADSO with ACO and BCO.

\begin{tabular}{|l|c|c|c|c|}
\hline Protocols & Latency & $\begin{array}{l}\text { Cognitive } \\
\text { transfer ratio }\end{array}$ & $\begin{array}{l}\text { Map transfer } \\
\text { ratio }\end{array}$ & $\begin{array}{l}\text { Average task } \\
\text { handling }\end{array}$ \\
\hline HMADSO & Low & High & High & Low \\
\hline ACO & High & Low & Low & Low \\
\hline BCO & High & Low & Low & \\
\hline
\end{tabular}

\subsection{HMADSO}

The analysis of HMADSO algorithm in terms of cooperative task handling brings improvement than $\mathrm{ACO}$ and $\mathrm{BCO}$. In FANETs increase in the quantity of UAV will result in decrease the number of tasks per UAV. Moreover, increase of UAV in network handle more tasks cooperatively but caused high latency. ACO offered lowest network cooperation time than BCO. In addition, HMADSO support good enough cooperation time than BCO. However, in HMADSO the latency of network operation is higher as compare to ACO but lower than BCO. Whenever the requirements are high packet delivery ratio, map transfer and the network redundancy is required low then HMADSO offered the best solution than other bio inspired algorithms but only latency issue remains in this optimization algorithm. Cognitive ratio results better in HMADSO with increase in number of nodes but has high network latency rather than utilization desert 
sparrow optimization and it is capable for resource distribution.

HMADSO has best performance associated with UAVs network parameters. This optimization is good for task distribution and cooperative rendezvous in FANET. Time and intricate of cooperative problems in FANETs are reduced by this algorithm. It is better in terms of task handling than ACO and
BCO. Only problem with HMADSO is its high latency which reduce the impact of such algorithm. If the network requirements are high packet ratio, map transfers and low redundancy of the network than HMADSO proved a better solution as compare to other techniques. Comparison characteristics of HMADSO with ACO, BCO is shows in table $\mathrm{V}$.

\section{Simulation Characteristics of Bio Inspired Schemes}

\begin{tabular}{|c|c|c|c|c|c|}
\hline \multicolumn{2}{|c|}{ Characteristics } & BeeAdHoc & $\begin{array}{l}\text { AntHocNet, } \\
\text { BeeAdHoc }\end{array}$ & $\begin{array}{c}\text { Moth-Ant } \\
\text { inspired routing }\end{array}$ & HMADSO \\
\hline \multirow{7}{*}{$\begin{array}{l}\text { Evaluated } \\
\text { objectives }\end{array}$} & Type & $\begin{array}{c}\text { Single } \\
\text { Objective }\end{array}$ & Single Objective & Single Objective & $\begin{array}{c}\text { Single } \\
\text { Objective }\end{array}$ \\
\hline & \multirow[t]{6}{*}{ Criteria } & $\begin{array}{c}\text { End-to-end } \\
\text { delay }\end{array}$ & End-to-end delay & End-to-end delay & Latency \\
\hline & & Throughput & Throughput & Throughput & \multirow[t]{2}{*}{$\begin{array}{c}\text { Packet } \\
\text { delivery ratio }\end{array}$} \\
\hline & & \multirow[t]{4}{*}{ Overhead } & \multirow[t]{4}{*}{ Overhead } & \multirow[t]{4}{*}{ Overhead } & \\
\hline & & & & & $\begin{array}{l}\text { Map transfer } \\
\text { ratio }\end{array}$ \\
\hline & & & & & $\begin{array}{c}\text { Network } \\
\text { cooperation } \\
\text { time } \\
\end{array}$ \\
\hline & & & & & $\begin{array}{c}\text { Task } \\
\text { handling }\end{array}$ \\
\hline \multirow{9}{*}{$\begin{array}{c}\text { Simulatio } \\
\mathbf{n} \\
\text { paramete } \\
\mathbf{r}\end{array}$} & $\begin{array}{c}\text { Operationa } \\
1 \text { area }\end{array}$ & $\begin{array}{c}1500 \mathrm{~m} * 1500 \\
\mathrm{~m}\end{array}$ & $1500 \mathrm{~m} * 1500 \mathrm{~m}$ & $\begin{array}{c}(300 \mathrm{~km} * 300 \mathrm{~km}) \\
\sim \\
(200 \mathrm{~km} * 200 \mathrm{~km})\end{array}$ & $\begin{array}{c}5000 * 5000 \mathrm{~m} \\
2\end{array}$ \\
\hline & $\begin{array}{c}\text { Simulation } \\
\text { runs }\end{array}$ & 10 & 10 & - & 100 \\
\hline & $\begin{array}{c}\text { Simulation } \\
\text { duration }\end{array}$ & $20 \mathrm{sec}$ & $20 \mathrm{sec}$ & - & $1000 \mathrm{sec}$ \\
\hline & UAVs & $10 \sim 50$ & $10 \sim 50$ & $25 \sim 50$ & $5 \sim 30$ \\
\hline & $\begin{array}{l}\text { Mobility } \\
\text { model }\end{array}$ & $\begin{array}{l}\text { Random } \\
\text { waypoint }\end{array}$ & Random waypoint & $\begin{array}{c}\text { Even and } \\
\text { singular for high } \\
\text { and low level } \\
\text { network } \\
\text { respectively } \\
\end{array}$ & - \\
\hline & $\begin{array}{c}\text { Node } \\
\text { velocity }\end{array}$ & $20 \sim 100 \mathrm{~m} / \mathrm{s}$ & $20 \sim 50 \mathrm{~m} / \mathrm{s}$ & $0.1 \sim 0.3 \mathrm{~km} / \mathrm{s}$ & $10 \mathrm{~m} / \mathrm{s}$ \\
\hline & $\begin{array}{c}\text { Traffic } \\
\text { type }\end{array}$ & CBR & CBR & - & CBR \\
\hline & $\begin{array}{c}\text { Signal } \\
\text { propagatio } \\
\mathrm{n} \text { model } \\
\end{array}$ & Friis & Friis & - & $\begin{array}{l}\text { Two-ray } \\
\text { ground }\end{array}$ \\
\hline & $\begin{array}{l}\text { MAC layer } \\
\text { protocol }\end{array}$ & 802.11 & 802.11 & TDMA-like & - \\
\hline
\end{tabular}


Shahzad Hameed (et al.), Nature Inspired Optimization Techniques, A review for FANETs

(pp. $40-58)$

\begin{tabular}{|c|c|c|c|c|c|}
\hline & $\begin{array}{c}\text { Data link } \\
\text { antenna }\end{array}$ & Omni & Omni & $\begin{array}{c}\text { Multi beam } \\
\text { directional }\end{array}$ & - \\
\cline { 2 - 6 } & $\begin{array}{c}\text { Antenna } \\
\text { coverage } \\
\text { range }\end{array}$ & $150 \sim 350 \mathrm{~m}$ & $\begin{array}{c}150 \sim 350 \\
\mathrm{~m}\end{array}$ & $<90 \mathrm{~km}$ & - \\
\cline { 2 - 6 } & $\begin{array}{c}\text { Transport } \\
\text { protocol }\end{array}$ & UDP & UDP & - & - \\
\cline { 2 - 6 } & Packet size & $512 \mathrm{~KB}$ & $512 \mathrm{~KB}$ & - & $1 \mathrm{~KB}$ \\
\hline
\end{tabular}

\section{Conclusion}

Routing and task allocation have unique requirement especially when deal with FANETs. Aspects may considerable to find the most efficient route, controlling the latency, ensuring the reliability, allowing the network to scale and ensure the Quality of Service. Design of suitable protocols and algorithms in FANET are the complex task due to its high mobility and dynamic changes topology. High node addition and removal behavior still see native protocols in FANET. In this review article we attempt to explain the research area based on biologically inspired algorithms. The reason to undertake this important survey is due to only few surveys focusing on naturally inspired field. We discuss six categories of protocols and see their applicability in FANETs. Further, different techniques of biologically inspired are discuss and see the extent to which they are suitable for FANETs. The routing and cooperation thru biological behaved algorithms provide the way towards the problem solution in FANET. The biological inspired routing algorithms BCO, ACO, moth and ant inspired routing and in addition hill Myna and desert Sparrow optimization prove to be efficient algorithms and overcome the problems facing in traditional protocols. Simulations based study will be the new consideration in future work with some other biological inspired algorithms. In present review article we focused on issues relating to routing protocols and task allocation based on bio inspired techniques.

\section{References}

[1] Bujari, Armir, Claudio E. Palazzi, and Daniele Ronzani. "FANET Application Scenarios and Mobility Models." In Proceedings of the 3rd Workshop on Micro Aerial Vehicle Networks, Systems, and Applications, pp. 43-46. ACM, 2017.

[2] Bekmezci, Ilker, Ismail Sen, and Ercan Erkalkan. "Flying ad hoc networks (FANET) test bed implementation." In Recent Advances in Space Technologies (RAST), 2015 7th International Conference on, pp. 665-668. IEEE, 2015.

[3] Mozaffari, Mohammad, Walid Saad, Mehdi Bennis, Young-Han Nam, and Mérouane Debbah. "A tutorial on UAVs for wireless networks: Applications, challenges, and open problems." IEEE communications surveys \& tutorials 21 , no. 3 (2019): 2334-2360.

[4] Gupta, Lav, Raj Jain, and Gabor Vaszkun. "Survey of important issues in UAV communication networks." IEEE Communications Surveys \& Tutorials 18, no. 2 (2016): 1123-1152.

[5] Karaboga, Dervis. An idea based on honey bee swarm for numerical optimization. Vol. 200. Technical reporttr06, Erciyes university, engineering faculty, computer engineering department, 2005.

[6] Dhurandher, Sanjay K., Sudip Misra, Puneet Pruthi, Shubham Singhal, Saurabh Aggarwal, and Isaac Woungang. "Using bee algorithm for peer-to-peer file searching in mobile ad hoc networks." 
Shahzad Hameed (et al.), Nature Inspired Optimization Techniques, A review for FANETs

(pp. $40-58)$

Journal of Network and Computer

Applications 34, no. 5 (2011): 14981508.

[7] Nugroho, Dwi Agung, Agi Prasetiadi, and Dong-Seong Kim. "Male-silkmothinspired routing algorithm for large-scale wireless mesh networks." Journal of Communications and Networks 17, no. 4 (2015): 384-393.

[8] Tareque, Md Hasan, Md Shohrab Hossain, and Mohammed Atiquzzaman. "On the routing in flying ad hoc networks." In Computer Science and Information Systems (FedCSIS), 2015 Federated Conference on, pp. 1-9. IEEE, 2015.

[9] Sahingoz, Ozgur Koray. "Networking models in flying ad-hoc networks (FANETs): Concepts and challenges." Journal of Intelligent \& Robotic Systems 74, no. 1-2 (2014): 513-527.

[10] Cheng, Chen-Mou, Pai-Hsiang Hsiao, H. T. Kung, and Dario Vlah. "Maximizing throughput of UAV-relaying networks with the load-carry-and-deliver paradigm." In Wireless Communications and Networking Conference, 2007. WCNC 2007. IEEE, pp. 4417-4424. IEEE, 2007.

[11] Jacquet, Philippe, Paul Muhlethaler, Thomas Clausen, Anis Laouiti, Amir Qayyum, and Laurent Viennot. "Optimized link state routing protocol for ad hoc networks." In Multi Topic Conference, 2001. IEEE INMIC 2001. Technology for the 21st Century. Proceedings. IEEE International, pp. 6268. IEEE, 2001.

[12] Alshbatat, Abdel Ilah, and Liang Dong. "Cross layer design for mobile ad-hoc unmanned aerial vehicle communication networks." In Networking, Sensing and Control (ICNSC), 2010 International Conference on, pp. 331-336. IEEE, 2010.

[13] Radu, Dan, Adrian Cretu, Benoît Parrein, Jiazi Yi, Camelia Avram, and Adina Aştilean. "Flying Ad Hoc Network for Emergency Applications Connected to a Fog System." In International

Conference on Emerging
Internetworking, Data \& Web Technologies, pp. 675-686. Springer, Cham, 2018.

[14] Perkins, Charles E., and Pravin Bhagwat. "Highly dynamic destination-sequenced distance-vector routing (DSDV) for mobile computers." In ACM SIGCOMM computer communication review, vol. 24, no. 4, pp. 234-244. ACM, 1994.

[15] Pei, Guangyu, Mario Gerla, and Tsu-Wei Chen. "Fisheye state routing: A routing scheme for ad hoc wireless networks." In Communications, 2000. ICC 2000. 2000 IEEE International Conference on, vol. 1, pp. 70-74. IEEE, 2000.

[16] Kaddoura, Maher, Barry Trent, Ranga Ramanujan, and Gregory Hadynski. "BGP-MX: Border gateway protocol with mobility extensions." In MILITARY COMMUNICATIONS CONFERENCE, 2011-MILCOM 2011, pp. 687-692. IEEE, 2011.

[17] Zhou, Qi, Wenzhe Gu, Jinglin Li, Qibo Sun, and Fangchun Yang. "A topology aware routing protocol based ADS-B system for aeronautical ad hoc networks." In Wireless Communications, Networking and Mobile Computing (WiCOM), 2012 8th International Conference on, pp. 1-4. IEEE, 2012.

[18] " DSR: The Dynamic Source Routing Protocol for Multi-Hop Wireless Ad Hoc Networks." [Online ] . Available : https ://www. ietf. org/rfc/rfc472 8 . txt. [Accessed: 23 -Mar-2016].

[19] Forsmann, J. Hope, Robert E. Hiromoto, and John Svoboda. "A time-slotted ondemand routing protocol for mobile ad hoc unmanned vehicle systems." In Unmanned Systems Technology IX, vol. 6561, p. 65611P. International Society for Optics and Photonics, 2007.

[20] Kuperman, Greg, Lenny Veytser, BowNan Cheng, Scott Moore, and Aradhana Narula-Tam. "A comparison of OLSR and OSPF-MDR for large-scale airborne mobile ad-hoc networks." In Proceedings of the third ACM workshop on Airborne 
networks and communications, pp. 1722. ACM, 2014.

[21] Billington, Jonathan, and Cong Yuan. "On modelling and analysing the dynamic MANET on-demand (DYMO) routing protocol." In Transactions on Petri Nets and Other Models of Concurrency III, pp. 98-126. Springer, Berlin, Heidelberg, 2009.

[22] Haas, Zygmunt J., Marc R. Pearlman, and Prince Samar. "The zone routing protocol (ZRP) for ad hoc networks." (2002).

[23] Wei, Yi, M. Brian Blake, and Gregory R. Madey. "An operation-time simulation framework for UAV swarm configuration and mission planning." Procedia Computer Science 18 (2013): 1949-1958.

[24] Park, Vincent. "Temporally-ordered routing algorithm (TORA) version 1 functional specification." Internet Draft, draft-ietf-manet-tora-spec-04. txt (2001).

[25] Flury, Roland, and Roger Wattenhofer. "Randomized 3D geographic routing." In INFOCOM 2008. The 27th Conference on Computer Communications. IEEE, pp. 834-842. IEEE, 2008.

[26] Liu, Cong, and Jie Wu. "Efficient Geometric Routing in Three Dimensional Ad Hoc Networks." In INFOCOM, pp. 2751-2755. 2009.

[27] Karp, Brad, and Hsiang-Tsung Kung. "GPSR: Greedy perimeter stateless routing for wireless networks." In Proceedings of the 6th annual international conference on Mobile computing and networking, pp. 243-254. ACM, 2000.

[28] Ko, Young-Bae, and Nitin H. Vaidya. "Location-Aided Routing (LAR) in mobile ad hoc networks." Wireless networks 6, no. 4 (2000): 307-321.

[29] Lidowski, Robert L., Barry E. Mullins, and Rusty O. Baldwin. "A novel communications protocol using geographic routing for swarming uavs performing a search mission." In Pervasive Computing and
Communications, 2009. PerCom 2009. IEEE International Conference on, pp. 17. IEEE, 2009.

[30] Pei, Guangyu, Mario Gerla, and Xiaoyan Hong. "LANMAR: landmark routing for large scale wireless ad hoc networks with group mobility." In Proceedings of the 1st ACM international symposium on Mobile ad hoc networking \& computing, pp. 11-18. IEEE Press, 2000.

[31] Basagni, Stefano, Imrich Chlamtac, Violet R. Syrotiuk, and Barry A. Woodward. "A distance routing effect algorithm for mobility (DREAM)." In Proceedings of the 4th annual ACM/IEEE international conference on Mobile computing and networking, pp. 76-84. ACM, 1998.

[32] Oh, Hyondong, Dario Turchi, Seungkeun Kim, Antonios Tsourdos, Lorenzo Pollini, and Brian White. "Coordinated standoff tracking using path shaping for multiple UAVs." IEEE Transactions on Aerospace and Electronic Systems 50, no. 1 (2014): 348363.

[33] Wan, Jiuqing, Liping Zhong, and Fan Zhang. "Cooperative localization of multi-UAVs via dynamic nonparametric belief propagation under GPS signal loss condition." International Journal of Distributed Sensor Networks 10, no. 2 (2014): 562380.

[34] Vincent, Patrick, and Izhak Rubin. "A framework and analysis for cooperative search using UAV swarms." In Proceedings of the 2004 ACM symposium on Applied computing, pp. 79-86. ACM, 2004.

[35] Kim, Do-Yup, and Jang-Won Lee. "Topology construction for flying ad hoc networks (FANETs)." In Information and Communication Technology Convergence (ICTC), 2017 International Conference on, pp. 153-157. IEEE, 2017.

[36] Leonov, Alexey V. "Application of bee colony algorithm for FANET routing." In Micro/Nanotechnologies and Electron Devices (EDM), 2016 17th International 
Conference of Young Specialists on, pp.

124-132. IEEE, 2016.

[37] Leonov, Alexey V. "Modeling of bioinspired algorithms AntHocNet and BeeAdHoc for Flying Ad Hoc Networks (FANETs)." In Actual Problems of Electronics Instrument Engineering (APEIE), 2016 13th International Scientific-Technical Conference on, vol. 2, pp. 90-99. IEEE, 2016.

[38] Hu, Lei, Fei Hu, and Sunil Kumar. "Moth-and Ant-Inspired Routing in Hierarchical Airborne Networks with Multi-Beam Antennas." IEEE Transactions on Mobile Computing (2018).

[39] Sharma, Vishal, D. G. Reina, and Rajesh Kumar. "HMADSO: a novel hill Myna and desert Sparrow optimization algorithm for cooperative rendezvous and task allocation in FANETs." Soft Computing (2017): 1-24. 